\title{
Competências do enfermeiro especialista em nefrologia
}

\author{
Competencies of the nurse specialist in nephrology \\ Competencias del enfermero especialista en nefrología
}

\author{
Nathalia Billo de Oliveira'; Frances Valéria Costa e Silva ${ }^{\text {II }}$ Luciana Guimarães Assad ${ }^{I I I}$
}

\begin{abstract}
RESUMO: Estudo quantiqualitativo, do tipo descritivo, objetivou discutir a compreensão dos enfermeiros sobre as atribuições e competências do especialista em nefrologia. Realizado no setor de hemodiálise, de um hospital universitário do município do Rio de Janeiro, entre julho e agosto de 2012, com 11 enfermeiros, por meio de entrevistas semiestruturadas. As entrevistas foram gravadas e transcritas. Os dados compuseram um banco em formato eletrônico, sendo agrupados em valores de mesma natureza e submetidos à técnica de análise de conteúdo. Os resultados demonstraram que a compreensão dos profissionais sobre suas atribuições e competências não estão bem definidas. Houve dificuldade em dar respostas consistentes e completas, fato que talvez possa ser atribuído à ausência de reflexão sobre o assunto. O estudo oferece subsídios para reflexão dos enfermeiros atuantes em hemodiálise acerca de suas atividades, buscando uma melhor atuação, uma prática baseada em competências e atitudes oriundas de um saber-pensar e um saber-fazer.
\end{abstract}

Palavras-Chave: Enfermagem; competência clínica; competência profissional; diálise renal.

\begin{abstract}
This quanti-qualitative, descriptive study discussed nurses' understanding of the duties and competencies of the specialist in nephrology. In July and August 2012, semi-structured interviews of 11 nurses were conducted at the hemodialysis unit of a university hospital in Rio de Janeiro city, recorded and later transcribed. The data were formatted as an electronic data base, grouped by values of the same kind and subjected to content analysis. The results showed that the professionals' understanding of their duties and competencies are not well defined. They had difficulty giving complete, consistent answers, which may perhaps be attributed to lack of thinking on the subject. The study provides food for thought for professional nurses in hemodialysis about their activities, with a view to improved performance, competence-based practice and postures derived from knowing how to think and how to do.
\end{abstract}

Keywords: Nursing; clinical competence; professional competence; renal dialysis.

RESUMEN: Estudio cuantitativo y cualitativo, del tipo descriptivo, con el objetivo de discutir la comprensión de los enfermeros en cuanto a las atribuciones y competencias del experto en nefrología. Ha sido realizado en la unidad de hemodiálisis de un hospital universitario, en el municipio de Rio de Janeiro, entre julio y agosto de 2012, con 11 enfermeros, a través de entrevistas semiestructuradas que fueron grabadas y transcritas. Los datos han compuesto un banco en formato electrónico, han sido agrupados en valores de misma naturaleza y sometidos a la técnica de análisis de contenido. Los resultados han demostrado que la comprensión de los profesionales acerca de sus atribuciones y competencias no está bien definida. Han tenido dificultad en dar respuestas consistentes y completas, un hecho que podría atribuirse a la falta de reflexión sobre el tema. El estudio ofrece subsidios para reflexión de los enfermeros que trabajan en hemodiálisis en cuanto a sus actividades, buscando un mejor rendimiento, una práctica basada en competencias y actitudes oriundas de un saber/ pensar y un saber/ hacer. Palabras Clave: Enfermería; habilidad clínica; competencia profesional; diálisis renal.

\section{INTRODUÇÃO}

O desenvolvimento da nefrologia como especialidade está associado às descobertas e ao aprimoramento das técnicas de diálise e transplante renal. Nesse sentido, os enfermeiros necessitaram aprender o manejo dos equipamentos e a melhor maneira de cuidar da clientela com insuficiência renal crônica ${ }^{1}$.

A fundação da Associação Brasileira de Enfermagem em Nefrologia (SOBEN), em 1983, marco para a es- pecialidade, teve por objetivo contribuir para "aperfeiçoar a assistência de enfermagem prestada aos indivíduos portadores de insuficiência renal"2:1. Porém a concessão de título de especialista em enfermagem em nefrologia teve início em 1996, após a criação do Departamento de Especialização e Títulos para Enfermeiros em Nefrologia (DETEN), responsável pelas titulações conferidas pela SOBEN, mediante provas de credenciamento.

IEnfermeira. Especialista em Nefrologia pela Faculdade de Enfermagem da Universidade do Estado do Rio de Janeiro. Enfermeira Supervisora no Centro de Nefrologia Mageense. Rio de Janeiro, Brasil, E-mail: nathaliabillo@yahoo.com.br.

IEnfermeira. Doutora em Saúde Coletiva. Professora da Universidade do Estado do Rio de Janeiro, Faculdade de Enfermagem, Departamento de Fundamentos de Enfermagem. Enfermeira da Nefrologia do Hospital Universitário. Rio de Janeiro, Brasil, E-mail: francesvcs@gmail.com.

IIIEnfermeira. Doutora em Enfermagem. Professora da Universidade do Estado do Rio de Janeiro, Faculdade de Enfermagem, Departamento de Fundamentos de Enfermagem. Chefe de Treinamento em Enfermagem do Hospital Universitário. Rio de Janeiro, Brasil, E-mail lgassad@gmail.com. 
Contribuiu também para o crescimento da especialidade a Resolução no 154/2004 da Diretoria Colegiada da Agência Nacional de Vigilância Sanitária, que trata da "necessidade de redefinir os critérios mínimos para o funcionamento de serviços de diálise" ${ }^{\text {3:1 }}$. A normatização estabelece a obrigatoriedade de enfermeiro especialista em nefrologia para assumir a responsabilidade técnica pelos serviços de enfermagem dos centros de diálise $e^{3}$.

Por se tratar de uma especialidade recente e com forte expansão, justifica-se o interesse de pesquisar as competências do enfermeiro na hemodiálise. $\mathrm{O}$ estudo desse tema implica o esforço de compreender as competências profissionais do enfermeiro especialista em nefrologia no desenvolvimento de suas ações e subsidia o planejamento de políticas de formação de profissionais durante a especialização.

A pesquisa teve como objeto as competências dos enfermeiros nefrologistas em hemodiálise e seu objetivo foi discutir a compreensão desses profissionais sobre as suas atribuições e competências.

\section{REVISÃO DE LITERATURA}

A pesquisa foi fundamentada nas concepções teóricas acerca do significado de competências profissionais do enfermeiro e na sua descrição relacionada à especialização em nefrologia, de acordo com a SOBEN ${ }^{1,2}$.

Do ponto de vista conceitual, o termo competência é compreendido como "capacidade de agir eficazmente em uma situação, apoiada em conhecimentos, mas sem limitar-se a eles" ${ }^{4: 7}$. É definido também como

um conjunto de saberes e capacidades que os profissionais incorporam por meio da formação e da experiência, somados à capacidade de integrá-los, utilizá-los e transferi-los em diferentes situações profissionais: ${ }^{5: 79}$.

Todo saber-fazer é uma competência, que é mais complexa por estar articulada com conhecimentos teóricos.

A partir do momento que o indivíduo faz o que deve ser feito sem sequer pensar, pois já o fez, não se fala mais em competências, mas sim em habilidades ou hábitos ${ }^{4: 27}$.

As habilidades são os resultados da construção das competências básicas que se consolidam na forma de habitus, ou o saber-fazer, que passam a ser, também, mobilizados na construção das competências profissionais ${ }^{5: 166}$.

As competências básicas são gerais e construídas pelo indivíduo para enfrentamento da vida, as intelectuais compreendem verdadeiros significados atribuídos às suas atitudes, que proporcionam a análise e solução de problemas, capacidade de tomada de decisão e adaptação, enquanto as profissionais são desenvolvidas durante a educação profissional de nível técnico até a graduação $0^{4-6}$.
As competências profissionais são entendidas como a capacidade de mobilizar, articular e colocar em prática valores, conhecimentos e habilidades necessários para o desempenho eficiente e eficaz de atividades no trabalho?

Outra competência desenvolvida é a do especialista, que acontece

quando o indivíduo é capaz de dominar com muita rapidez e segurança as situações mais comuns, por dispor de esquemas de ação, que, com um esforço razoável de reflexão, mobiliza rapidamente seus conhecimentos para enfrentar situações inéditas:2:26.

A SOBEN descreve as competências dos profissionais de enfermagem nefrologistas agrupando-as em quatro áreas, a saber: administrativa, assistencial, educativa e de pesquisa. Cada profissional é orientado por um perfil e uma postura fornecidos pela formação de sua categoria ${ }^{8}$.

\section{Metodologia}

Trata-se de uma pesquisa quantiqualitativa, do tipo descritiva. Foi realizada no setor de hemodiálise de um hospital universitário no Rio de Janeiro, caracterizada como unidade de referência de tratamento de doenças renais graves e centro de formação profissional na área de saúde.

Teve como sujeitos os profissionais de enfermagem de nível superior, lotados no setor, no período entre julho e agosto de 2012. Participaram da pesquisa 11, de um total de 12 enfermeiros inseridos no serviço. A ausência de um dos sujeitos derivou de sua impossibilidade de responder a entrevista. Para a manutenção do anonimato, foram atribuídos números aos participantes das entrevistas (E1, E2,... E11).

Para a coleta de dados, foi utilizada a entrevista semiestruturada e um instrumento fechado para a identificação das atividades e responsabilidades gerais desses profissionais no setor. A entrevista foi gravada em meio digital e as falas foram transcritas para tratamento posterior.

Os dados transcritos constituíram textos submetidos à técnica de análise de conteúdo, com agregação, síntese, ordenação e classificação dos dados, categorizados por semelhanças de conteúdos, repetição e ausências9.

Um documento, disponibilizado pela SOBEN, que discorre sobre as competências dos enfermeiros em nefrologia ${ }^{8}$, foi utilizado como matriz para a categorização dos dados. Foram construídas quatro categorias: competências assistenciais, administrativas, educativas e de pesquisa.

A pesquisa respeitou os princípios da Resolução $n^{\circ}$ 196/1996, dando oportunidade aos profissionais de optarem em participar ou não do estudo. Todos os participantes assinaram o Termo de Consentimento Livre 
e Esclarecido. A pesquisa foi submetida ao Comitê de Ética em Pesquisa da Universidade do Estado do Rio de Janeiro/ SR2, sendo aprovada em 28/06/2012 com protocolo $\mathrm{n}^{\circ}$ 026.3.2012.

\section{Resultados e Discussão}

\section{Perfil dos participantes}

grupo estudado foi predominantemente do sexo feminino, $7(63,6 \%)$, possui idade média de 39,3 anos $(+5,9)$, apresentando extremos de 28 a 48 anos. O tempo médio de formação foi de 9,1 anos $(+5,7)$ e variou de 3 a 19 anos. Com relação à instituição formadora, 6(54,5\%) participantes eram egressos de instituição privada e $5(45,5 \%)$ de universidades públicas.

Com relação à pós-graduação, 9 (81,8\%) tinham formação adicional, enquanto 2(18,2\%) negaram tal condição. Entre aqueles com pós-graduação, 6(67\%) tinham a especialização lato sensu, 2(22\%) especialização e residência e $1(11 \%)$ residência. Entre os especialistas, existiam 7(78\%) com formação em nefrologia.

O tempo de atuação no serviço variou de um mês a cinco anos incompletos, sendo 4(36,4\%) com menos de 11 meses, 4(36,4\%) entre 12 e 47 meses, e 3(27,3 \%) com 48 meses ou mais. A experiência prévia na área existia na maioria do grupo estudado - 7(63,6\%) -, com tempo variando entre dois e 15 anos.

Dos profissionais, $7(63,6 \%)$ se consideram especialistas em nefrologia, entretanto poucos associam esta condição a sua titulação formal, mas ao fato de possuir experiência prática, além do tempo de trabalho. Considerando as justificativas para não se considerar especialista na área, as respostas variaram entre não possuir título e trabalhar pouco tempo no setor. A SOBEN determina, para obter o título de especialista por prova, a comprovação de cinco anos de experiência como enfermeiro em nefrologia e aprovação na avaliação teórica por eles organizada ou cursar uma pós-graduação lato -sensu em nefrologia com cadastro na associação ${ }^{2}$.

\section{Competências assistenciais}

A maior parte das competências assistenciais listadas pela $\mathrm{SOBEN}^{8}$ foi contemplada nos depoimentos, seja como atribuição ou propriamente como competência, o que demonstra o forte vínculo do grupo com as atividades associadas ao cuidado ao cliente. Foi possível perceber que os enfermeiros mobilizam saberes por meio de esquemas de ação durante o cuidado, possibilitando que os conhecimentos utilizados gerem competências específicas para o profissional na nefrologia, o que só é possível quando são traduzidas em atos. Os esquemas de ação são importantes porque ligam os sujeitos ao meio, uma vez que estão associados à percepção, avaliação e tomada de decisão durante o cuidado ${ }^{10}$.
As atividades assistenciais contemplaram o cuidado direto, a orientação e educação aos pacientes e familiares, contribuindo para que o enfermeiro crie espaços de interação ativos possibilitando a individualização do cuidado.

A orientação com o cliente e família é uma atividade desenvolvida por $8(72 \%)$ profissionais, sendo que $3(28 \%)$ admitem que a dinâmica do trabalho à noite, a função de gerência e a rotina intensa os levam a se distanciar dessa função, limitando-se a orientar quando abordados.

A educação está dirigida prioritariamente a clientes recém-admitidos, tendo por objetivo esclarecer dúvidas sobre doença renal crônica e terapia renal substitutiva, além de reduzir o medo da máquina de hemodiálise. Houve referência ao uso de recursos educativos como folhetos e palestras, quando possível. Um profissional mencionou que investiga outros problemas de saúde que podem estar acometendo esses clientes, não se detendo exclusivamente à doença renal crônica. Nesse cenário, emerge a importância da produção de um saber coletivo que se traduz na autonomia e emancipação dos sujeitos e da sua família ${ }^{11}$.

O cuidado e manipulação dos cateteres e fístulas estiveram presentes em todas as falas, denotando a importância atribuída pelos enfermeiros à manutenção dos acessos em hemodiálise, pois sem eles o procedimento não pode ser realizado.

Cuidados com as fístulas e cateteres no dia a dia. (E6)

Puncionar as fístulas, um enfermeiro, que não é da hemodiálise, não tem competência para isto, ele deve conhecer para evitar problemas. (E7)

A interpretação de exames laboratoriais, bem como a identificação juntamente com o médico, indivíduo e família sobre o melhor método dialítico para aquele cliente é expressa como competência assistencial pela $\mathrm{SOBEN}^{8}$, existindo 2(18\%) referências a estas ações. Neste contexto, é pertinente assinalar o conhecimento e a autonomia profissional como elemento que confere profissionalização a um trabalho ${ }^{12}$.

Discussão sobre os exames dos pacientes, avaliando a adequação de diálise e intervir quando achar necessário. Manter uma comunicação, melhor interação com os médicos para ter maior ciência sobre o quadro atual dos pacientes [...] (E6)

Ver os resultados de exames para saber se estão bons ou não, avaliar o paciente que dialisa se pode passar pra outro tipo de terapia. (E3)

O especialista em nefrologia deve ser capaz de prevenir, identificar e tratar complicações apresentadas pelos clientes antes, durante e após o procedimento dialítico, de acordo com a $\mathrm{SOBEN}^{8}$. Os enfermeiros entendem que perceber intercorrências ao longo do procedimento dialítico é possível a partir 
de: experiência prática - 9(56\%) -, conhecimento científico - 5(32\%) -, e atenção e agilidade em perceber situações de risco - 2(12\%).

$\mathrm{Na}$ ação assistencial do enfermeiro, existem seis áreas de conhecimentos práticos que podem ser analisadas para se compreender as competências desenvolvidas. Destacam-se as máximas, que são as instruções fornecidas pelos especialistas, que não fazem sentido para qualquer outro, a menos que a pessoa já tenha conhecimento profundo da situação ou que disponha de larga experiência na área ${ }^{13}$.

Competências adicionalmente descritas versam sobre o conhecimento aprofundado, o pensamento crítico, o direcionamento do cuidado e o embasamento teórico nas ações. Admitem que o especialista em nefrologia possui conhecimento aprofundado sobre as doenças renais e as terapias de substituição renal e, por meio disso, seriam capazes de direcionar melhor o cuidado, bem como ter embasamento teórico nas ações.

Nesse contexto, ressalta-se o Parecer CNE/CP $n^{\circ}$ 29/2002 do MEC o qual informa que competências incluem: intuir, pressentir e arriscar, com base em experiências anteriores e conhecimentos, habilidades e valores articulados e mobilizados para resolver os desafios da vida profissional, que exigem respostas sempre novas, originais, criativas e empreendedoras ${ }^{14}$.

Algumas competências descritas pela SOBEN ${ }^{8}$ não foram mencionadas - consultas de enfermagem, a orientação e supervisão dos procedimentos de desinfecção de equipamentos e de reprocessamento dos dialisadores. A ausência de referências não pode ser entendida como ações não realizadas, mas sim como o não reconhecimento da ação como competência. Também podem ser reconhecidas como administrativas, incluídas na compreensão da ação do enfermeiro como supervisor.

\section{Competências administrativas}

"Historicamente o enfermeiro é um agente cuidador, porém, na contemporaneidade, vem se firmando como administrador da assistência"15:554. As bases ideológicas e teóricas da administração em enfermagem contemplam quatro domínios do ato de administrar: o planejamento, a tomada de decisão, a supervisão e a auditoria ${ }^{16}$. Dentre esses domínios, os entrevistados referiram três deles, sendo a auditoria o domínio não citado. Outras respostas foram obtidas como: registro no prontuário, provimento de material, organização, fiscalização e controle da qualidade da água de diálise.

Organização do serviço, tomada de decisão, gerenciamento, atribuições de assistência designadas exclusivamente ao enfermeiro. (E4)

Supervisão da equipe; interagir com os doentes; provimento de material e insumos, operar máquinas e prestar assistência. (E6)

\section{[...] registro de intercorrências em prontuário eletrônico} ou não, que facilita a comunicação e permite saber o que aconteceu com o paciente nos outros dias. (E3)

O trabalho dos enfermeiros deve estar fundamentado na capacidade de tomar decisões de modo a garantir um resultado efetivo, sem desperdiçar recursos. Para este fim, devem possuir habilidades para avaliar, sistematizar e decidir a conduta mais apropriada ${ }^{7}$, sobressaindo à liderança como competência a ser desenvolvida ${ }^{17}$.

Das competências administrativas propostas pela $\mathrm{SOBEN}^{8}$, não foram mencionadas a promoção de reuniões com a equipe; a elaboração de protocolos de enfermagem, a minimização de eventos adversos; a implantação, planejamento, organização, execução e avaliação da sistematização da assistência de enfermagem (SAE), entre outros itens propostos. Neste contexto, é pertinente citar a construção de uma matriz de competência profissional do enfermeiro ${ }^{18}$ explorando suas competências básicas em um cenário de especialidade, que chama atenção para a necessidade de um sistema de linguagem padronizado para definir competência. Portanto, a ausência de expressão dos enfermeiros sobre competências que lhe são próprias pode ser relacionada à falta de uma linguagem que permita mensurar com clareza o que se quer dizer.

Ainda no espaço das competências administrativas, cabe destaque a um estudo, também explorando habilidades e competências de enfermeiros em cenário especializado, que tem como achado relevante a dimensão gerencial nos depoimentos dos sujeitos ${ }^{19}$.

\section{Competências educativas}

As respostas dos enfermeiros relacionadas a esta categoria consideram a educação como um processo direcionado aos clientes e aos profissionais. Ressaltase que tais atividades de educação ao cliente e família também são mencionadas pelo documento da SOBEN como competências assistenciais. Os enfermeiros reconhecem como atribuições educativas as atividades de educação com os clientes e a coordenação e preceptoria de residentes e estagiários e citam como competências o esclarecimento de dúvidas da terapia, produção de material explicativo, ensino, recepção de pessoas em treinamento e transmissão de conhecimento.

\footnotetext{
Mais do que administrar o setor, a gente tinha que vincular este especialista a trabalhar com educação do paciente, a orientação à doença dele, tornar esse momento dele um pouco mais tranquilo, com isso você ia ter uma assistência muito melhor, ia conhecer esse paciente de outra forma [...]. (E11)

A SOBEN descreve a promoção de atividade de educação dos clientes e familiares como competência do enfermeiro especialista em nefrologia, relacionada à melhora da qualidade de vida, a qual pode ser efetuada por meio de orientações e da formulação de manuais educativos adequados a sua realidade socia ${ }^{18}$.
} 
Cabe ao enfermeiro, em primeiro lugar, recepcionar o cliente nefropata e tentar esclarecer o mar de dúvidas em que geralmente ele vem mergulhado, podendo fazer isto com várias estratégias que devem ser somadas, desde a abordagem direta por meio de entrevistas e, também, pela produção de materiais informativos impressos, ou pela formulação de mídias eletrônicas e grupos aproveitando tempo e espaço disponíveis para que se possa ouvir e educar o cliente. (E11)

Outras competências educativas estabelecidas pela SOBEN implicam: proporcionar condições para o aprimoramento dos profissionais de enfermagem atuantes na área, mediante cursos e estágios em instituições afins; participar e estimular a presença em eventos científicos, de atualização na área; participar da elaboração de programas de estágios, treinamento e desenvolvimento de profissionais de enfermagem, nos diferentes níveis de formação, bem como supervisionar pessoal de enfermagem em estágios na nefrologia ${ }^{8}$. Tais atividades também estavam presentes nos relatos dos entrevistados.

As respostas obtidas nesta categoria demonstram que os entrevistados reconhecem as atividades de educação com clientes, com os profissionais iniciantes e sua própria atualização, seja em forma de treinamento ou de educação continuada, como parte de suas atribuições e competências. Apesar disso, argumentam que sua atualização é limitada por razões particulares e as ações dirigidas a clientes e profissionais iniciantes são muitas vezes suprimidas por não fazerem parte de um processo de trabalho planejado e controlado, ficando a critério da iniciativa de cada enfermeira.

\section{Competências de pesquisa}

Os sujeitos do estudo não contemplaram, nos discursos obtidos, competências relacionadas à pesquisa. A SOBEN descreve a pesquisa como importante competência profissional, apresentada como estabelecimento de "[...] relações técnico-científicas com as unidades afins, desenvolvendo estudos investigacionais e de pesquisas [...]", bem como a participação "[...] da avaliação de produtos novos em nefrologia [...]" ${ }^{\text {::2 }}$. Estes achados indicam ser necessário reforçar o incentivo ao desenvolvimento de investigações, buscando evidências científicas para a prática do enfermeiro na nefrologia.

\section{ConClusão}

Compreende-se a competência como a capacidade de agir com eficácia em determinadas situações, embasada por conhecimentos e habilidades que podem ser incorporados à clínica por meio da formação e da prática, somadas à capacidade de integrá-los e utilizálos em suas atitudes. Portanto, pode-se dizer que a competência é integrada por três elementos principais: o conhecimento que seria o saber; a habilidade caracterizada pelo saber-fazer e a atitude do querer-fazer.
Em relação à compreensão dos profissionais sobre suas atribuições e competências, pode-se destacar que eles não as têm bem definidas. Houve dificuldade em dar respostas consistentes e completas, fato que talvez possa ser atribuído à falta de reflexão sobre a sua prática profissional ou, ainda, à limitação do estudo em função de sua aplicação em apenas um serviço de nefrologia.

O estudo proporcionou reflexão e entendimento sobre as competências e atribuições dos especialistas de enfermagem em nefrologia na área de hemodiálise. Questionando enfermeiros especialistas, na prática, sobre seus esquemas e saberes mobilizados, durante o cuidado, foi um dos meios de esclarecer as competências profissionais a serem adquiridas em um sistema de formação.

\section{REFERÊNCIAS}

1.Lima EX, Santos I, organizadoras. Atualização de enfermagem em nefrologia. Rio de Janeiro: SOBEN; 2004.

2.Associação Brasileira de Enfermagem em Nefrologia [site de internet]. História da sociedade. [citado em 31 out 2014] Disponível em: http://www.soben.org.br/historiada-sociedade

3.Agência Nacional de Vigilância Sanitária (Br). Resolução- RDC no 154, de 15 de junho de 2004. [citado em 31 out 2014]. Disponível em: http://www.anvisa.gov.br/ hotsite/segurancadopaciente/documentos/rdcs/RDC\%20 N\%C2\%BA\%20154-2004.pdf

4.Perrenoud P, Magne BC. Construir as competências desde a escola. Porto Alegre (RS): Artmed; 1999.

5.Ramos MN. A pedagogia das competências: autonomia ou adaptação? São Paulo: Cortez; 2001.

6.Instituto Nacional de Educação e Pesquisas Educacionais (Br). Documento básico do exame nacional do ensino médio 2000. [citado em 31 out 2014] Disponível em: www. fisica.ufmg.br/ menfis/programa/Docbasico2000.doc

7.Ministério da Educação (Br). Resolução CEB n4, de 8 de dezembro de 1999. [citado em 31 out 2014] Disponível em: http://portal.mec.gov.br/cne/arquivos/pdf/rceb04_99.pdf. 8.Associação Brasileira de Enfermagem em Nefrologia. Departamento de títulos de especialistas de enfermagem em nefrologia. Formação de recursos humanos. Belo Horizonte (MG): SOBEN; 2011.

9.Bardin L. Análise de conteúdo. Lisboa (Pt): Edições $70 ; 2007$.

10.Perrenoud P, Murad F, Alessandrini CD. Formando professores profissionais: quais estratégias? quais competências? Porto Alegre (RS): Artmed; 2001.

11. Machado MFAS, Monteiro EMLM, Queiroz DT, Vieira NFC, Barroso MGT. Integralidade, formação de saúde, educação em saúde e as propostas do sus: uma revisão conceitual. Ciênc saúde coletiva. 2007; 12: 335-42. 12.Versa GLS, Matsuda LM. Satisfação profissional da equipe de enfermagem intensivista de um hospital de ensino. Rev enferm UERJ. 2014; 22: 409-15.

13.Benner PE. From novice to expert: excellence and power in clinical nursing practice. Upper Saddle River (NJ): Prentice Hall; 2001. 
14.Ministério da Educação (Br). Resolução n³, 18 de dezembro de 2002. [citado em 29 out 2013] Disponível em: http://portal.mec.gov.br/cne/arquivos/pdf/cp29.pdf. 15.Montezelli JH, Peres AM. Competência gerencial do enfermeiro: conhecimento publicado em periódicos brasileiros. Cogitare enfermagem. 2009; 14:553-8

16.Sanna MC. A estrutura do conhecimento sobre administração em enfermagem. Rev Bras Enferm. 2007; 60: 336-8.

17.Silva VLS, Camelo SHH. A competência da liderança em enfermagem: conceitos, atributos essenciais e o papel do enfermeiro líder. Rev enferm UERJ. 2013 21:533-9. 18.Holanda FL, Marra CC, Cunha ICKO. Construção da matriz de competência profissional do enfermeiro em emergências. Acta Paul Enferm. [Scielo Scientific Eletronic Library Online]. 2014 [citado em 01 nov 2014]; 27:373-9. Disponível em: http://www.scielo.br/scielo.php?script=sci arttext\&pid $=$ S0103-21002014000400014\&lng = pt. http:// dx.doi.org/10.1590/1982-0194201400062

19.Prado RT, Dias SM, Castro EAB. Competências e habilidades para atuação do enfermeiro em bancos de olhos. Texto contexto - enferm. [Scielo-Scientific Eletronic Library Online]. 2014 [citado em 01 nov 2014]; 23: 47-55. Disponível em: http://www.scielo.br/scielo.php?script=sci arttext\&pid $=$ S0104-07072014000100047\&lng $=$ pt. http://dx.doi.org/10.1590/S0104-07072014000100006. 